\title{
Die Tuberkulosesituation in Deutschland 2001 und 2002
}

\author{
B. Hauer ${ }^{1}$ \\ B. Brodhun ${ }^{2}$ \\ D. Altmann² \\ D. Sagebiel ${ }^{1}$ \\ W. Haas $^{2}$ \\ R. Loddenkemper ${ }^{1}$
}

Tuberculosis in Germany in 2001 and 2002

\section{Zusammenfassung}

Durch die Einführung des Infektionsschutzgesetzes im Jahr 2001 und die damit verbundene Erfassung erweiterter Meldevariablen hat sich die Kenntnis über die epidemiologische Tuberkulosesituation in Deutschland verbessert. Im Jahr 2001 wurden dem Robert Koch-Institut 7515, im Jahr 20027684 Neuerkrankungen an behandlungsbedürftiger Tuberkulose gemeldet, entsprechend einer Inzidenz von 9,1 bzw. 9,3/100000 Einwohner (beides Stand: 1.10.2003). Der geringe Anstieg der Neuerkrankungen im Jahr 2002 begründet sich aller Wahrscheinlichkeit nach auf einer Untererfassung durch die Einführung des neuen Meldesystems im Jahr 2001, zumal die Inzidenz für 2003 mit 8,7/100 000 (7184 Erkrankungen) den zuvor langjährig rückläufigen Trend fortsetzt. Sowohl im Jahr 2001 als auch 2002 wurde bei $42 \%$ der an behandlungsbedürftiger Tuberkulose Erkrankten ein Geburtsland außerhalb Deutschlands angegeben, die Inzidenz liegt bei Menschen mit ausländischer Staatsangehörigkeit durchschnittlich etwa fünfmal so hoch wie bei deutscher Staatsangehörigkeit. Unverändert ist in über $80 \%$ der Fälle die Lunge das am häufigsten betroffene Organ. In 4230 (2001) bzw. 4267 (2002) Fällen handelt es sich dabei um eine offene, das heißt bakteriologisch bestätigte, Lungentuberkulose mit potenzieller Ansteckungsgefahr für die unmittelbare Umgebung. Die Resistenzraten sind bei der einheimischen Bevölkerung stabil. Bei Menschen aus Herkunftsländern mit Resistenzproblemen spiegelt sich die dortige Situation wider. Mehr als zwei Drittel der Tuberkulosepatienten werden in Deutschland zu irgendeinem Zeitpunkt stationär betreut. Die Behandlungserfolge für 2001 erreichen mit

\section{Abstract}

The implementation of the new law on infectious diseases in 2001 and the resulting notification of more detailed variables has led to a better knowledge about the epidemiology of tuberculosis (TB) in Germany. 7,515 new cases of active tuberculosis were notified to the Robert Koch Institute in 2001, and 7,684 cases in 2002, corresponding to an incidence of 9.1 (2001) and 9.3 (2002) per 100,000 inhabitants (as per 1 October, 2003). The slight increase in new cases in 2002 is most likely due to undernotification in 2001 because of the new system, considering that the previous decline over many consecutive years is continued according to the incidence for $2003(8.7 / 100,000 ; n=7,184)$. In 2001 as well as in $200242 \%$ of new TB patients were born outside Germany, and those of foreign nationality have a five-fold higher incidence on the average than German citizens. The lung remains the most affected organ (80\%). 4,230 (2001) and 4,267 (2002) were cases of open, i.e., bacteriologically confirmed, pulmonary tuberculosis potentially contagious to their immediate contacts. The resistance rates are stable in the indigenous population, while patients coming from countries with high resistance rates reflect the situation in their countries of origin. Over two thirds of TB patients in Germany are hospitalized at some point during their disease. The treatment results $(78 \%$ on the average in 2001) do not reach the WHO target of $85 \%$. Approximately $7 \%$ of TB patients in Germany still die because of this infectious disease.

Institutsangaben

${ }^{1}$ Deutsches Zentralkomitee zur Bekämpfung der Tuberkulose (DZK), Lungenklinik Heckeshorn, Berlin

${ }^{2}$ Robert Koch-Institut, Berlin

Korrespondenzadresse

Prof. Dr. med. R. Loddenkemper, Generalsekretär · Deutsches Zentralkomitee zur Bekämpfung der Tuberkulose · Lungenklinik Heckeshorn · Zum Heckeshorn 33 · 14109 Berlin .

E-mail: loddheck@zedat.fu-berlin.de

Eingang: 23. Dezember 2004 · Angenommen: 3. Januar 2005

Bibliografie

Pneumologie 2005; 59: 264-269 @ Georg Thieme Verlag KG Stuttgart • New York

DOI 10.1055/s-2004-830212

ISSN 0934-8387 
durchschnittlich 78\% nicht das von der WHO angestrebte Ziel von $85 \%$. Etwa $7 \%$ der Tuberkulosepatienten sterben in Deutschland auch heute noch infolge dieser Infektionskrankheit.

\section{Einleitung}

Die Tuberkulose (TB) ist neben Malaria und HIV die weltweit häufigste Infektionskrankheit, es sterben mehr Menschen daran als an jeder anderen behandelbaren Infektionskrankheit. Die Weltgesundheitsorganisation (WHO) nimmt an, dass ein Drittel der Menschheit mit M. tuberculosis infiziert ist, von diesen entwickeln etwa $5-10 \%$ im Laufe ihres Lebens eine behandlungsbedürftige Tuberkulose. Global wird von einem jährlichen Anstieg der Tuberkulosefälle von 0,4\% ausgegangen. Jeden Tag erkranken weltweit etwa 20000 Menschen, 5000 sterben täglich an einer Tuberkulose. Insgesamt geht die WHO von 8 - 9 Millionen Neuerkrankungen und 2 Millionen Todesfällen an Tuberkulose pro Jahr aus. Einer Eradikation der Tuberkulose stellen sich vor allem die Problematik der TB/HIV-Koinfektion sowie das zunehmende Auftreten resistenter Tuberkuloseerreger entgegen. Hohe Tuberkuloseinzidenzen finden sich hauptsächlich im südlich der Sahara gelegenen Afrika sowie in Südostasien. Für Deutschland besonders bedeutsam ist die Lage in den Staaten der ehemaligen Sowjetunion (Neue Unabhängige Staaten, NUS), welche mit hohen Tuberkuloseinzidenzen und erschreckenden Resistenzraten zu kämpfen haben - Folgen der eingeschränkten Gesundheitsversorgung und der sozialen Veränderungen nach Beendigung des kalten Krieges [10]. Die höchste Inzidenz dieser Region, mit weiter steigender Tendenz, ist 2002 in Kasachstan mit 178/100000 registriert worden. In der Russischen Föderation scheint sich gemäß der offiziellen Zahlen möglicherweise eine Trendwende abzuzeichnen, da nach einem Inzidenzgipfel im Jahr 2000 von 97/100 000 für 2001 eine Inzidenz von 92 und für 2002 von 89/100 000 gemeldet wurde. Diese Entwicklung ist jedoch mit Vorsicht zu interpretieren, da beispielsweise die Auswirkungen der rasch ansteigenden HIV-Infektionsraten in den NUS [11] zum jetzigen Zeitpunkt noch nicht abzusehen sind.

In Deutschland ist, übereinstimmend mit der Mehrheit der westund zentraleuropäischen Länder [3,12], seit Jahrzehnten ein rückläufiger Trend zu beobachten. Die Neuerungen des Infektionsschutzgesetzes (IfSG) [1], welches im Januar 2001 das Bundesseuchengesetz abgelöst hat, haben zu einer verbesserten Surveillance der Tuberkulosesituation in Deutschland geführt. In den Vorjahren basierten die Antworten auf wichtige epidemiologische Fragestellungen, wie beispielsweise die Resistenzsituation und den Behandlungserfolg, vornehmlich auf der DZK-Studie zur Epidemiologie der Tuberkulose, welche unter Leitung von Dr. Michael Forßbohm/Gesundheitsamt Wiesbaden in den Jahren 1996 - 2000 in Zusammenarbeit mit rund 50\% der deutschen Gesundheitsämter durchgeführt wurde [4]. Viele der hierbei gewonnenen Erfahrungen wurden in der Ausgestaltung des IfSG berücksichtigt. Die Meldepflichten für Tuberkulose wurden präzisiert und die zu erhebenden Daten an internationale Vorgaben angepasst. Es wurde eine einzelfallbasierte Erfassung auf der Grundlage einer einheitlichen, durch das Robert Koch-Institut (RKI) herausgegebenen, Falldefinition [8] sowie eine zentrale
Übermittlung der Fälle auf nationaler Ebene etabliert. Die den Gesundheitsämtern gemeldeten Erkrankungs- oder Todesfälle werden anhand der Falldefinition bewertet und über die zuständigen Landesgesundheitsbehörden auf elektronischem Wege an das RKI übermittelt.

Dank dieses modernen Meldesystems verfügt Deutschland auch im internationalen Vergleich über eine sehr gute TuberkuloseSurveillance. Die routinemäßige Erfassung des Behandlungserfolges war ausschlaggebend dafür, dass Deutschland im Sinne der WHO nun zu den DOTS-Ländern (directly observed treatment, short-course) gezählt wird [12].

Die im Folgenden aufgeführten Zahlen beruhen im Wesentlichen auf den Daten des RKI [7]. Die Auswertungen beziehen sich dabei auf die Fälle, welche nach IfSG die Kriterien der Referenzdefinition erfüllten. Das bedeutet, nach Bewertung des Gesundheitsamtes entsprechen die Meldungen den klinischen, klinisch-labordiagnostischen oder klinisch-epidemiologischen (das heißt aufgrund eines epidemiologischen Zusammenhangs mit einem gesicherten Fall) Kriterien der Falldefinition des RKI. Entscheidend für das Vorliegen einer Tuberkulose ist, dass eine Behandlung eingeleitet wurde oder - falls der Patient vor Diagnosestellung verstarb - eingeleitet worden wäre. Ein direkter Vergleich mit dem Datenmaterial der Vorjahre, welches vom Statistischen Bundesamt erhoben und als jährliche Informationsberichte vom Deutschen Zentralkomitee zur Bekämpfung der Tuberkulose zusammengestellt und veröffentlicht wurde, ist aufgrund der nun neu angewandten Referenzdefinition nicht ohne weiteres möglich.

\section{Die Tuberkulosesituation in Deutschland 2001 und 2002}

Die nachfolgenden Daten beziehen sich auf die übermittelten Fälle gemäß Referenzdefinition, die bis zum 1.10. 2003 vorlagen.

Im Jahr 2001 wurden dem RKI 7515, im Jahr 20027684 Neuerkrankungen an Tuberkulose gemeldet, entsprechend einer Inzidenz von 9,1 bzw. 9,3/100 000. Die Anzahl der 2003 gemeldeten Tuberkulosen beläuft sich auf 7184 Fälle (Inzidenz 8,7/100 000, Stichtag 1.10. 2004 [6]). Die Angaben für das Jahr 2001 müssen unter Vorbehalt gesehen werden, da es durch die Umstellung des Meldesystems aller Wahrscheinlichkeit nach zu einer Untererfassung gekommen ist. Der vermeintlich leichte Anstieg der Fallzahlen in 2002 ist daher dennoch als fortlaufend rückläufiger Trend zu werten, zumal auch die Zahlen für 2003 einen weiteren Rückgang zeigen. Der leichte Anstieg der Tuberkuloseinzidenzen in der ausländischen Bevölkerung Anfang der 1990er-Jahre ist wahrscheinlich überwiegend durch die damaligen Migrationsbewegungen erklärbar (Abb.1). Die folgende Darstellung konzentriert sich im Detail auf die für das Jahr 2002 erhobenen epidemiologischen Daten im Vergleich zum Vorjahr. 


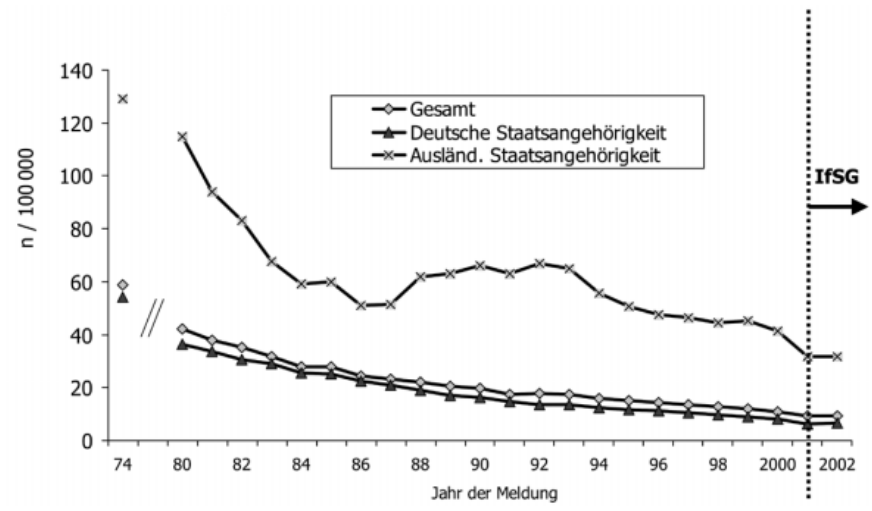

Abb. 1 Die Entwicklung der Tuberkuloseinzidenz in Deutschland 1974-2002 (vor 1991 nur alte Bundesländer, ab 2001 Meldung nach IfSG).

\section{Geburtsland}

(von $\mathrm{n}=6964 / 7684$ )

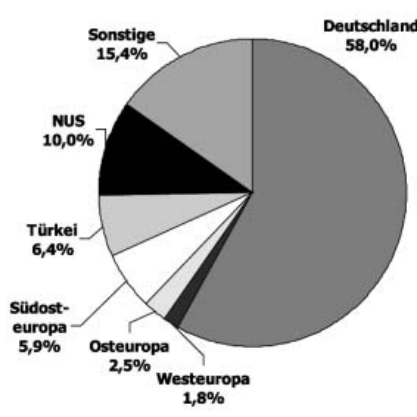

Staatsangehörigkeit (von $n=7365 / 7684$ )

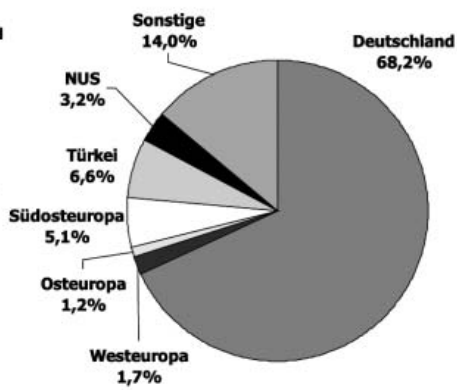

Abb. 2 Herkunft der Tuberkulosepatienten in Deutschland 2002.

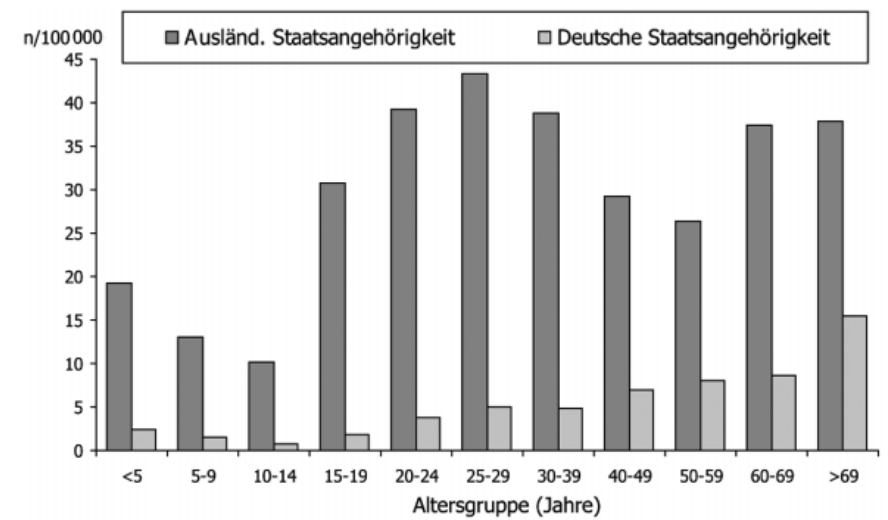

Abb. 3 Tuberkuloseinzidenz nach Altersgruppe und Staatsangehörigkeit $2002(\mathrm{n}=7359)$.

\section{Staatsangehörigkeit und Geburtsland}

Für 6964 der 2002 gemeldeten Fälle an behandlungsbedürftiger Tuberkulose (90,6\%; 2001 : 6691 entsprechend 89,0\%) lagen Angaben zum Geburtsland, für 7365 (95,8\%; 2001 : 7062 entsprechend $94,0 \%$ ) zur Staatsangehörigkeit vor. In beiden Jahren lag das Geburtsland in $42 \%$ der Fälle außerhalb Deutschlands. Im Vergleich dazu betrug der Anteil der Patienten mit einer ausländischen Staatsangehörigkeit lediglich 31,8\% (2002) bzw. 32,7\% (2001), woraus folgt, dass mit alleiniger Erhebung der Staatsbür- gerschaft der Anteil der Migranten unter den Tuberkulosepatienten in Deutschland unterschätzt würde. In Abb. 2 sind die Herkunftsländer nach Geburtsland und Staatsangehörigkeit vergleichend dargestellt. 10,8\% (2002) bzw. 9,1\% (2001) der Personen, bei denen sowohl Angaben zur Staatsangehörigkeit als auch zum Geburtsland vorlagen, hatten die deutsche Staatsangehörigkeit, waren aber im Ausland geboren. Von den 741 Fällen im Jahr 2002 stammten 460 dieser Patienten aus den NUS ${ }^{1}$, dabei überwiegend aus der Russischen Föderation (50,2\%) und aus Ka$\operatorname{sachstan}(40,4 \%)$.

Die Tuberkuloseinzidenz in der Patientengruppe mit ausländischer Staatsangehörigkeit betrug 31,9/100000 (2001: $31,7 / 100000$ ), sie lag damit knapp fünfmal so hoch wie bei der einheimischen Bevölkerung (2002: 6,7; 2001: 6,3/100000). Abb. 3 zeigt die Inzidenzen nach Altersgruppen und Staatsangehörigkeit für das Jahr 2002. Vergleiche mit den Jahren vor 2001 werden dadurch eingeschränkt, dass vor Einführung des IfSG alle Fälle mit unbekannter Staatsangehörigkeit den nicht-deutschen Fällen zugeordnet wurden, wodurch dieser Anteil möglicherweise überschätzt wurde, wohingegen die Inzidenz aufgrund der 2002 und 2001 nicht berücksichtigten Fälle unbekannter Staatsangehörigkeit (2002: 4,2\%; 2001: 7,9\%) möglicherweise unterschätzt wird.

Bei Personen mit deutscher Staatsangehörigkeit stieg ab einem Alter von 15 Jahren die Inzidenz mit zunehmendem Alter kontinuierlich an. Im Kindesalter fand sich die höchste Inzidenz bei den unter 5-Jährigen. Bei den Erkrankten mit ausländischer Staatsangehörigkeit gab es drei Inzidenzgipfel: bei Kindern unter fünf Jahren (19,3/100 000), im Alter zwischen 25 und 29 Jahren $(43,3 / 100000)$ und bei Personen über 69 Jahren (37,9/100 000) (Abb.3). Die Fallzahl bei Personen über 69 Jahren mit ausländischer Staatsangehörigkeit ist jedoch klein $(\mathrm{n}=89)$, weshalb diese, im Gegensatz zu den Senioren mit deutscher Staatsangehörigkeit $(n=1483)$, epidemiologisch nicht ins Gewicht fallen.

\section{Organbeteiligung und bakteriologischer Status}

Angaben zur Organbeteiligung lagen 2002 für 96,1\% der Fälle vor (7388/7684). Wie im Vorjahr war die Lunge mit knapp 81\% $(n=5950)$ die häufigste Lokalisation. Eine Lungentuberkulose wurde dabei signifikant häufiger bei Männern angegeben ( $85,0 \%$ vs. 73,9\%; $p<0,001$ ), entsprechend überwog bei den extrapulmonalen Formen der Anteil weiblicher Erkrankter. Die Lungentuberkuloseinzidenz der Frauen betrug 5,4/100000 Einwohner, die der Männer 11,1/100 000. Bei im Ausland geborenen Erkrankten war der Anteil von Lungentuberkulosen signifikant geringer als bei deutschen Patienten (76,8\% vs. 83,6\%; $\mathrm{p}<0,001$ ).

Bei den 5950 gemeldeten Lungentuberkulosen (80,6\%) betrug der Anteil offener Lungentuberkulosen 71,7\% (4267 Fälle). Dabei handelte es sich in 2030 Fällen (34,1\% der Lungentuberkulosen) um eine hoch ansteckende Form, bei der mikroskopisch säure-

\footnotetext{
${ }^{1}$ NUS: Russische Föderation, Kasachstan, Ùkraine, Belarus, Kirgistan, Tadschikistan, Moldavien, Estland, Lettland, Litauen, Aserbaidschan, Georgien, Turkmenistan, Usbekistan, Armenien
} 


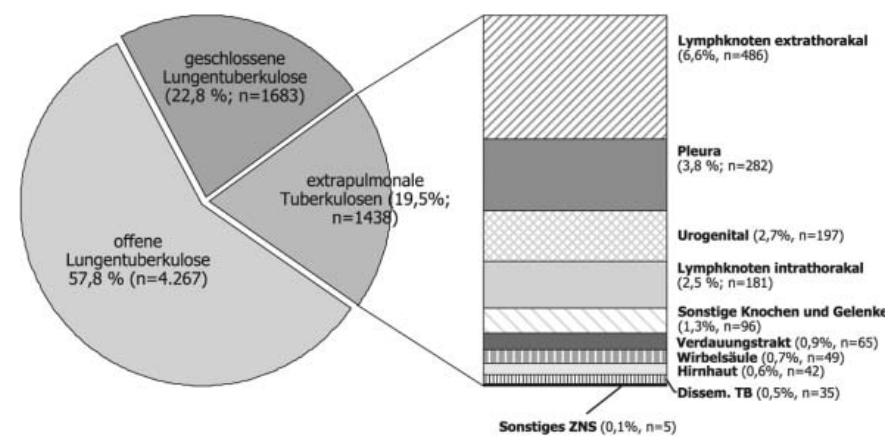

Abb. 4 Verteilung der Tuberkuloseformen in Deutschland 2002 $(n=7388)$.

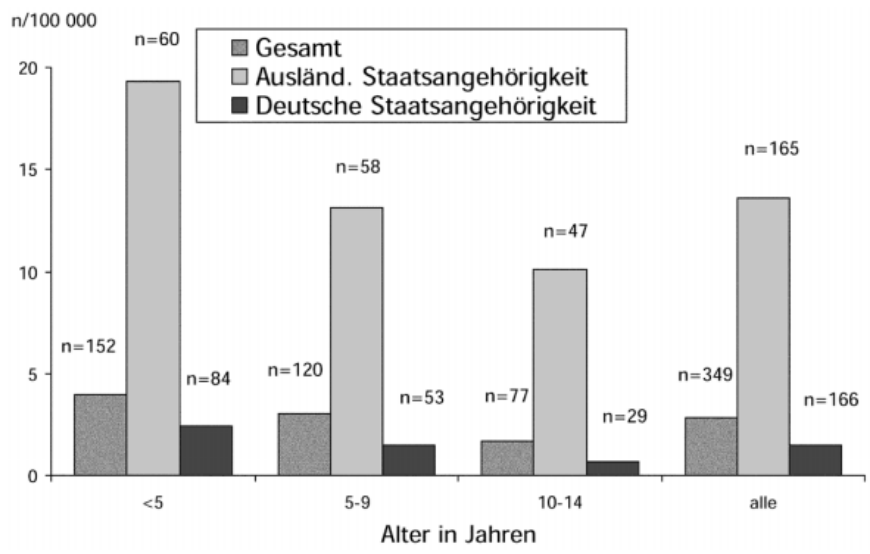

Abb. 5 Kindertuberkulose in Deutschland 2002 nach Staatsangehörigkeit.

feste Stäbchen im Sputum nachgewiesen werden konnten. Abb. 4 gibt einen Gesamtüberblick über die Verteilung auf die einzelnen Tuberkuloseformen für 2002. Angaben zum Organ lagen dabei in insgesamt 7388 Fällen vor.

\section{Die Tuberkulose im Kindesalter}

In der statistischen Erfassung werden Personen zum Kindesalter gerechnet, die das 15 . Lebensjahr noch nicht vollendet haben.

In Deutschland erkrankten 2002 insgesamt 349 Kinder an einer behandlungsbedürftigen Tuberkulose, dies entspricht einer Inzidenz von 2,8/100 000 Kindern. Im Vergleich dazu wurden 2001 insgesamt 291 Neuerkrankungen, entsprechend einer Inzidenz von 2,3/100 000, im Kindesalter gemeldet. Dieser scheinbare Anstieg dürfte jedoch in erster Linie mit den bereits angesprochenen Problemen bei der Umstellung des Meldesystems durch die Einführung des IfSG mit einer daraus resultierenden Untererfassung für 2001 zusammenhängen. Die weiteren Angaben konzentrieren sich daher auf die Meldestatistik für 2002. Mädchen erkrankten geringfügig häufiger als Jungen (52,1\%, Inzidenz 3,0/100 000 vs. $47,9 \%$, Inzidenz 2,6/100000). Die höchste Inzidenz fand sich in der Altersgruppe der Kinder unter 5 Jahren, mit einem Maximum bei den 2- und 3-Jährigen (4,8 bzw. 5,8/100 000).

Von den Kindern, bei denen Angaben zur Staatsangehörigkeit vorlagen ( $n=331 ; 94,8 \%$ ), besaßen 165 Kinder (49,8\%) eine aus- ländische und 166 (50,2\%) die deutsche Staatsangehörigkeit (Abb.5). Interessant ist auch hier, dass dabei das Geburtsland bei 208 Kindern (64,6\%) mit „Deutschland“ und nur bei 114 Kindern $(35,4 \%)$ mit „Ausland“ angegeben wurde. Für Kinder mit ausländischer Staatsangehörigkeit lag das Risiko, an einer Tuberkulose zu erkranken, je nach Altersgruppe 8- bis 14,5-mal so hoch wie für deutsche Kinder. Bei letzteren betrug die durchschnittliche Inzidenz 1,5/100 000, bei ausländischer Staatsangehörigkeit 13,6/100 000 (Abb.5). Die Inzidenz der Kinder ausländischer Staatsangehörigkeit stieg dabei im Vergleich zum Vorjahr für alle Altersgruppen an (unter Vorbehalt, s.o.), bei den deutschen Kindern fiel der Inzidenzanstieg dagegen deutlich geringer aus.

Von den Kindern, bei denen Angaben zur Lokalisation der Tuberkulose vorlagen $(n=336,96,3 \%)$, handelte es sich in 247 Fällen $(73,5 \%)$ um eine Lungentuberkulose, wobei die Verteilung deutscher und ausländischer Staatsangehörigkeit nahezu identisch war. Die übrigen Tuberkulosemanifestationen betrafen die Lymphknoten (13,1\% intrathorakal; $n=44$; extrathorakal $7,4 \%$, $\mathrm{n}=25$ ). Danach lagen in absteigender Reihenfolge Tuberkulosen des Verdauungstrakts $(1,5 \%, n=5)$, sonstiger Knochen und Gelenke $(1,2 \%, n=4)$, der Pleura $(1,2 \%, n=4)$, der Hirnhaut $(0,9 \%$, $\mathrm{n}=3)$, disseminierte Tuberkulosen $(0,6 \%, \mathrm{n}=2)$, Tuberkulosen des sonstigen ZNS $(0,3 \%, n=1)$ und der Wirbelsäule $(0,3 \%, n=1)$ vor. Aufgrund der niedrigen Tuberkuloseinzidenzen in Deutschland und entsprechenden Nutzen-Risiko-Abwägungen wird von der Ständigen Impfkommission am RKI (STIKO) die BCG-Impfung in Deutschland seit 1998 nicht mehr empfohlen [9].

\section{Klinikaufenthalt}

Bei Jugendlichen und Erwachsenen über 14 Jahren lagen in 7227 Fällen $(98,6 \%)$ Informationen zu dieser Fragestellung vor. Im Durchschnitt wurden 70,0\% (64,0-74,6\%) der Erkrankten zu irgendeinem Zeitpunkt stationär betreut, ohne besondere geschlechtsspezifische Unterschiede. Der Anteil der in Deutschland geborenen Patienten war etwas höher als derjenige der im Ausland geborenen (73,1\% vs. 70,4\%), nach Altersstandardisierung lassen sich jedoch keine Unterschiede mehr feststellen.

Im Kindesalter kam es im Jahr 2002 bei rund 70\% (Schwankungsbreite je nach Altersgruppe 58,3 - 77,8\%) der Kinder (237/338) zu irgendeinem Zeitpunkt der Diagnose bzw. Therapie zu einem Klinikaufenthalt. Eine stationäre Aufnahme war im Kleinkindesalter etwas häufiger als bei älteren Kindern.

\section{Anlass der Diagnosestellung}

Für das Erfassungsjahr 2002 lagen in 6513 der 7684 Fälle Informationen zum Anlass der Diagnose vor. Die Ergebnisse sind in Tab. 1 dargestellt. Die aktive Fallfindung war bei im Ausland Geborenen signifikant häufiger als bei in Deutschland Geborenen (22,4\% vs. 10,8\%; p < 0,001). Die Zahlen belegen, dass ein nicht unwesentlicher Teil der Tuberkulosen in Deutschland durch aktive Fallfindung bei Risikogruppen entdeckt wird, hierbei spielen insbesondere die Umgebungsuntersuchung und Untersuchungen nach §36 IfSG eine bedeutende Rolle. 
Tab. 1 Anlass der Diagnosestellung $2002(n=6513 / 7684)$

\begin{tabular}{ll}
\hline Anlass & TB-Fälle \\
\hline passive Fallfindung & $\begin{array}{l}5412 \\
\text { Symptome }\end{array}$ \\
$42=0,6 \%$ Obduktion/postmortale Untersuchungen \\
\hline aktive Fallfindung & $1059=16,3 \%$ \\
in Risikogruppen & $430=6,6 \%$ Umgebungsuntersuchung \\
400 & $=6,1 \%$ Screening nach $\S 36$ IfSG \\
105 & $=1,6 \%$ Überwachung gesunder Befundträger \\
101 & $=1,6 \%$ Gemeinschaftseinrichtung \\
23 & $=0,4 \%$ Aufenthaltsberechtigung
\end{tabular}

\section{Resistenzlage}

Bis zur Einführung des IfSG wurde die Resistenzlage für Deutschland auf der Basis der vom Nationalen Referenzzentrum für Mykobakterien in Borstel (NRZ), vom Arbeitskreis Mykobakterien (AKM) und im Rahmen der DZK-Studie 1996-2000 erhobenen Daten eingeschätzt. Nach IfSG werden Resistenzen nun miterhoben und stehen somit bundesweit auf Einzelfallbasis zur Verfügung.

Tab. 2 gibt eine Übersicht der Resistenzentwicklung in Deutschland und beinhaltet die im Rahmen der DZK-Studie erhobenen Daten sowie die durch das RKI ausgewerteten Meldungen. Dabei liegen die nach IfSG erhobenen Resistenzraten deutlich über denen der DZK-Studie. Nach unserer Einschätzung kann daraus nicht ohne weiteres ein Anstieg der Resistenzraten abgeleitet werden, zumal die unterschiedlichen Erhebungsarten (Sentinelbzw. Surveillance-System) einen direkten Vergleich der RKI- mit den DZK-Resistenzraten nur eingeschränkt zulassen. Zu den Multiresistenzraten (Multiresistenz $=$ multidrug resistance/ MDR, d. h. Resistenz gegenüber mind. Isoniazid u. Rifampicin) ist noch anzumerken, dass es sich - mit Blick auf eine statistische Aussagekraft - um verhältnismäßig kleine Fallzahlen (2002:90 Fälle) handelt, aber im europäischen Vergleich sind diese Absolutzahlen durchaus hoch. Die RKI-Daten zeigen, übereinstimmend mit der DZK-Studie, dass Resistenzen häufiger bei Vorbehandelten zu finden sind, das heißt bei Fällen, in deren Vorgeschichte eine mindestens einmonatige antituberkulotische The- rapie durchgeführt wurde. So lag beispielsweise die MDR-Rate im Falle einer Vorbehandlung mit 8,8\% sechs mal so hoch wie bei nicht-vorbehandelten Patienten mit 1,5\%.

Während die Resistenzsituation bei Patienten deutscher Herkunft auf niedrigem Niveau stabil ist, spiegelt sich die weltweite Epidemiologie in höheren Resistenzraten bei im Ausland geborenen Patienten wider. Für Deutschland spielen hier insbesondere Migranten aus den Staaten der ehemaligen Sowjetunion eine wichtige Rolle. So ergab die DZK-Studie bei Patienten aus den Neuen Unabhängigen Staaten (NUS) für den Zeitraum 1996 - 2000 eine jegliche Resistenz (HRES) von im Durchschnitt 29,9\% und eine MDR-Rate von 9,9\% [4]. Die RKI-Daten (2002) ergeben für diese Patientengruppe eine jegliche Resistenz in 37,8\% der Fälle und eine MDR-Rate von 12,4\%.

Eine kürzlich veröffentlichte Arbeit aus dem Forschungszentrum Borstel [5] beschreibt unter den im Zeitraum 1995-2001 dort eingegangenen MDR-Stämmen ( $\mathrm{n}=451)$ einen Clusteranteil von 49\%. Die Autoren folgern, dass es in einem durchaus bedeutenden Teil der Fälle zu Übertragungen von MDR-Stämmen in Deutschland kommt, bemerken jedoch zu Recht einschränkend, dass der Anteil der Fälle, bei denen eine Infektion bereits im Herkunftsland mit den dort endemischen Stämmen erfolgte, unklar ist.

\section{Behandllungsergebnis}

Aufgrund der langen Behandlungsdauer einer Tuberkulose liegen Resultate zum Ausgang einer Behandlung erst mit einer entsprechend großen Verzögerung vor. Für das Jahr 2001 waren bis zum Stichtag am 1. 10. 2003 für 5986 (79,4\%) der Fälle Angaben zum Behandlungsergebnis verfügbar. In 78,0\% der Fälle wurde die Tuberkulose erfolgreich behandelt (vollständige antituberkulotische Therapie mit oder ohne negativen bakteriologischen Befund). Der Therapieerfolg zeigt eine deutliche Altersabhängigkeit, so wird bei über 69-Jährigen nur ein Wert von etwa $60 \%$ erreicht. Interessant ist zudem, dass - bis auf das Kindesalter - der Behandlungserfolg bei Frauen signifikant besser ausfällt als bei Männern.

Insgesamt 1312 Patienten (22,0\%) schlossen die Therapie nicht erfolgreich ab. Ein wichtiger Grund hierfür war mit 61,9\% $(n=813)$ das Versterben, insbesondere älterer Menschen, vor Ab-

Tab. 2 Resistenz in Deutschland 1996-2002

\begin{tabular}{|c|c|c|c|c|c|c|c|c|c|}
\hline \multirow{3}{*}{ DZK-Studie } & 1997 & 2690 & 5,8 & 1,7 & 3,9 & 1,4 & 2,6 & 1,4 & 7,7 \\
\hline & 1998 & 2696 & 6,5 & 1,8 & 5,4 & 1,5 & 2,8 & 1,4 & 9,3 \\
\hline & 1999 & 2849 & 5,5 & 1,8 & 5,1 & 1,7 & 2,5 & 1,4 & 8,3 \\
\hline \multirow[t]{2}{*}{ RKI } & 2001 & 4389 & 7,0 & 2,4 & 6,5 & 2,2 & 2,6 & 2,3 & 9,8 \\
\hline & 2002 & 4489 & 8,0 & 2,3 & 7,6 & 2,1 & 2,2 & 2,0 & 11,0 \\
\hline
\end{tabular}

Quellen: 28. Informationsbericht DZK, RKI-Bericht 2004. H = Isoniazid, R = Rifampicin, S = Streptomycin, E = Ethambutol, Z = Pyrazinamid 


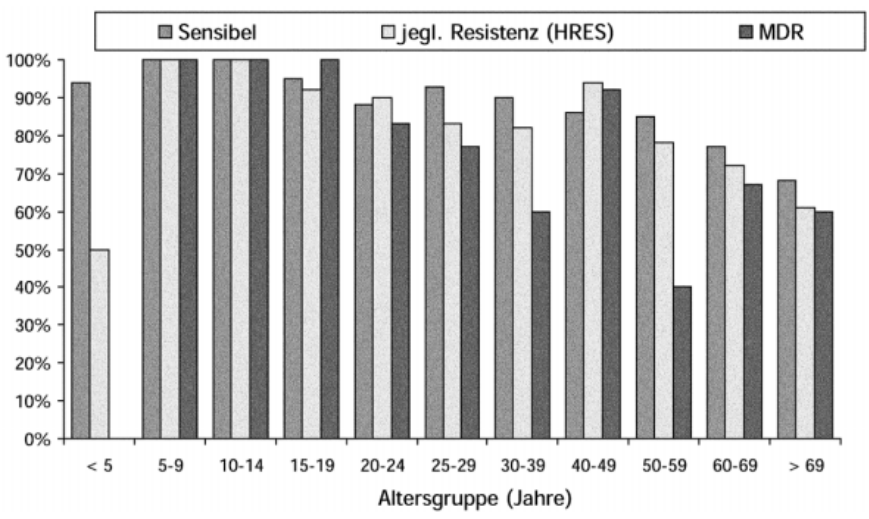

Abb. 6 Behandlungserfolg 2001 in Abhängigkeit von der Erregerresistenz nach Altersgruppen ( $n=3393$; voll sensibel: 80,7\% erfolgreicher Abschluss; MDR: 65,1\%).

schluss der Therapie (davon war bei 251 Fällen die Tuberkulose auch die Todesursache). Das Erreichen der von der WHO geforderten Therapieerfolgsrate von $85 \%$ ist für Industrienationen wie Deutschland, mit einem relevanten Teil an Alterstuberkulosen (bei deutschen Patienten) und damit an Sterbefällen vor bzw. unter Therapie, sehr schwierig. 326 Patienten $(24,8 \%)$ hatten die Therapie zum Stichtag noch nicht abgeschlossen, 160 (12,2\%) hatten die Behandlung abgebrochen, und in 13 Fällen (1,0\%) wurde ein Versagen der Therapie gemeldet.

Bei Betrachtung des Behandlungsergebnisses nach Geburtsland zeigt sich ein signifikant höherer Behandlungserfolg bei den im Ausland geborenen Patienten (durchschnittlich $86,2 \%$ vs. $74,0 \%$, $\mathrm{p}<0,001$ ).

Entscheidenden Einfluss auf einen erfolgreichen Therapieausgang hatte, neben dem Alter, auch das Vorliegen von Medikamentenresistenzen. So betrug die Therapieerfolgsrate bei Patienten mit multiresistenten Stämmen lediglich 65,1\% im Vergleich zu 80,7\% Behandlungserfolg bei Patienten mit Infektion durch sensible Erreger. Abb. 6 zeigt das Behandlungsergebnis in Abhängigkeit von der Erregerresistenz für die einzelnen Altersgruppen.

\section{Die Mortalität der Tuberkulose}

Die bisherige Erfassung in der Todesursachenstatistik wertete lediglich die Daten des zum Tode führenden Grundleidens und nicht der zum Tode führenden Erkrankung aus. Mit dem IfSG werden erstmals Daten zur Häufigkeit von Todesfällen an Tuberkulose erfasst. Das Merkmal „Tod an Tuberkulose“ wird sowohl im Rahmen der Basisdaten aller meldepflichtigen Erkrankungen als auch im Rahmen der Erfassung des Behandlungsergebnisses erhoben. Als Behandlungsergebnis wird zwischen dem Tod an Tuberkulose und dem Tod durch andere Ursachen während einer Erkrankung an Tuberkulose unterschieden. Dabei ist jedoch zu berücksichtigen, dass nicht für alle Fälle Angaben zum Behandlungsergebnis vorliegen. So waren für 2001 lediglich in 79,4\% der Fälle (Stichtag 1.10. 2003) Angaben zum Behandlungsergebnis verfügbar. Es kann davon ausgegangen werden, dass etwa 7\% der an behandlungsbedürftiger Tuberkulose Erkrankten an dieser versterben.
Fazit

Die Tuberkulose ist in Deutschland - trotz insgesamt rückläufiger Neuerkrankungsraten - nach wie vor eine hochrelevante Infektionskrankheit. Im Zeitalter der Globalisierung wirkt sich die zunehmende Migration, insbesondere aus Ländern mit hohen Tuberkulose- und HIV-Prävalenzen sowie besorgniserregenden Medikamentenresistenzraten, auch auf die Tuberkulosesituation in Deutschland aus. Diese Entwicklungen müssen in Diagnostik, Therapie und Prävention ausreichende Berücksichtigung finden. Ein Nachlassen in den Bemühungen um eine effiziente und zeitgemäße Tuberkulosekontrolle in Deutschland könnte mittelund langfristig schwerwiegende Folgen haben. Vielmehr erfordert die Tuberkulose aufgrund der rückläufigen Erfahrung mit diesem Krankheitsbild mehr denn je unsere Aufmerksamkeit in der Lehre und im medizinischen Alltag. Denn eine zeitnahe Diagnose und adäquate Therapie infektiöser Patienten sowie die kompetente Zusammenarbeit zwischen klinischem Sektor und öffentlichem Gesundheitsdienst sind und bleiben die Grundpfeiler einer erfolgreichen Tuberkulosekontrolle. Zur effizienten Bekämpfung der Tuberkulose bedarf es der genauen Kenntnis der epidemiologischen Situation und der Umsetzung in Diagnostik und Therapie. Gute Daten, auf deren Basis auch die Gesundheitsämter ihrer Verantwortung nachkommen können, setzen voraus, dass die behandelnden Ärzte ihrer Meldepflicht zuverlässig und so umfassend wie möglich nachkommen.

\section{Literatur}

${ }^{1}$ Bales S, Baumann HG, Schnitzler N. Infektionsschutzgesetz. Kommentar und Vorschriftensammlung. 2. überarbeitete Auflage. Kohlhammer-Verlag, 2003

${ }^{2}$ Corbett EL, Watt CJ, Walker N et al. The Growing Burden of Tuberculosis. Global Trends and Interactions with the HIV Epidemic. Arch Int Med 2003; 163: 1009-1021

${ }^{3}$ Euro TB. Surveillance of Tuberculosis in Europe. Report on tuberculosis cases notified in 2001. Paris, 2003 http://www.eurotb.org

${ }^{4}$ Forßbohm M. Studie des DZK zur Epidemiolgie der Tuberkulose. Abschlussbericht 1996-2000 (26333 Fälle). In: Deutsches Zentralkomitee zur Bekämpfung der Tuberkulose: 28. Informationsbericht 2004 (erhältlich beim DZK).

${ }^{5}$ Kubica T, Rüsch-Gerdes S, Niemann S. The Bejjing genotype is emerging among multidrug-resistant Mycobacterium tuberculosis strains from Germany. Int J Tuberc Lung Dis 2004; 8: 1107- 1113

${ }^{6}$ Robert Koch-Institut. Tuberkulose in Deutschland 2003. Epidemiologisches Bulletin 2004; 44: 375 - 377

${ }^{7}$ Robert Koch-Institut. Bericht zur Epidemiologie der Tuberkulose in Deutschland für 2002. Berlin: Mercedes Druck, 2004

${ }^{8}$ Robert Koch-Institut. Falldefinitionen des Robert Koch-Instituts zur Übermittlung von Erkrankungs- oder Todesfällen und Nachweisen von Krankheitserregern. Berlin: Druckpunkt, 2004

${ }^{9}$ Robert Koch-Institut. Ständige Impfkommission am Robert Koch-Institut (STIKO): Impfempfehlungen. Epidemiologisches Bulletin 1998; 15: $109-112$

${ }^{10}$ Sagebiel D, Brendel A, Forßbohm M. Gefährliche Zunahme multiresistenter Tuberkulosen in der ehemaligen Sowjetunion. Pneumologie 2002; 56: 7-10

${ }^{11}$ UNAIDS. The changing HIV/AIDS epidemic in Europe and Central Asia. UNAIDS 0418E, April 2004

${ }^{12}$ WHO. Report 2004. Global Tuberculosis Control: Surveillance, Planning, Financing. WHO/HTM/TB/2004.331. 2004

${ }^{13}$ WHO/IUATLD. Anti-tuberculosis drug resistance in the world. Report No. 3. (2004). WHO/CDS/TB/2004.xxx, Im Druck 